\title{
La experiencia de confianza de los niños en proceso de educación musical: una relación del cuidado'
}

\author{
Jéssica Marcela Quintero Jurado²
}

Recibido: 10-08-2017

Aceptado: 18-10-2017

\section{RESUMEN}

Esta investigación tuvo como objetivo comprender las experiencias de confianza, como sentimiento moral, en el proceso de educación musical de niños y niñas entre 9 y 11 años de la Escuela Musical Amadeus de la ciudad de Medellín. Se adoptó el método fenomenológico-hermenéutico. Para la selección de los participantes se realizó un muestreo por fases. Las técnicas que se utilizaron fueron fotohistoria, fotolenguaje, entrevista en profundidad y grupo focal como estrategia de triangulación. Se encontró que la relación de confianza posee unas características que la consolidan como una relación de cuidado, pues posibilita el aprendizaje y el acceso a nuevas experiencias, promueve y facilita la regulación emocional, es considerada por los niños y las niñas como una relación empática y comprensiva, y está asociada a una expectativa de incondicionalidad.

Palabras clave: confianza, cuidado, sentimientos morales, educación musical. 


\title{
The experience of trust of children in musical education: a caring relationship
}

\begin{abstract}
The objective of this research is to understand the trusting experiences, as a moral feeling, in the process of musical education of children between the ages of 9 and 11 years old from Amadeus Musical School in the city of Medellin. The phenomenological-hermeneutic approach was opted. For the selection of the participants, a sampling was realized by stages. The techniques used were picture-story, photo-language, the profound interview, and the focal group as a triangulation strategy. It was found that the trust relationship poses characteristics that consolidate it as a caring relationship, since it allows teaching and access to new experiences, promotes and facilitates the emotional regulation, it's considered by the children as an empathetic and comprehensive relationship, and it is associated to unconditional support.
\end{abstract}

Keywords: Trust, care, moral feelings, musical education.

\section{Introducción}

Los contextos de educación musical se reconocen como escenarios de socialización y una posibilidad para el desarrollo de las capacidades humanas. En este sentido, al ser espacios de interacción con otros, que además permiten la ampliación del mundo relacional, juegan un papel fundamental en el desarrollo moral y en la educación emocional de los niños, y son fundamentales en la comprensión de sus trayectorias de desarrollo moral. Además, estos espacios se van consolidando como comunidades afectivas, de las que los niños forman parte y en las cuales circulan sentimientos morales como la confianza.

La confianza es conceptualizada en este estudio como un sentimiento moral desde la teoría cognitivo-evaluadora de las emociones (Nussbaum, 2008), que concibe las emociones como elementos esenciales de la 
inteligencia humana, entendiendo que las emociones contienen en sí mismas conciencia de valor e importancia sobre objetos externos en tanto elementos en el esquema de los propios objetivos o proyectos importantes, por lo que se consideran como parte esencial del sistema de razonamiento ético, pues contienen juicios que pueden ser verdaderos o falsos, y pautas malas y buenas para las elecciones éticas. En definitiva, las emociones desempeñan un papel importante en el pensamiento acerca de lo bueno y lo justo.

Al respecto, Camps (2011) afirma que la construcción y mantenimiento de la confianza requiere emprender diversas estrategias, que tienen todas como común denominador:

el cumplimiento de las expectativas puestas en uno, o dicho de otro modo, el sentido de responsabilidad (...) No defraudar, ser capaz de mantener las expectativas creadas, hacerse cargo de ellas, es el requisito para que no se pierda la esperanza ( $p$. 199).

De esta manera, se entiende el sentimiento de confianza como fruto de un proceso de gestación, consolidación, afianzamiento y mantenimiento, que se reconoce como "un trabajo no sólo largo y profundo, sino de paciencia, minuciosidad" (Langer, 2016, p. 121), pero que también incluye su posibilidad de fractura, vulneración y pérdida. Este proceso que se presenta en la vida pública de los ciudadanos, es gestado inicialmente en los vínculos íntimos, pues, como lo afirma Camps (2011), es el espacio privado en el que puede gestarse mejor la confianza, una confianza que aporte seguridad y autoestima imprescindibles para fortalecer la autonomía, y pensar y decidir por sí mismo.

El proceso de gestación de la confianza comienza en la primera infancia consolidándose en los primeros vínculos con los cuidadores. Así, la generación de la confianza es posible sólo mediante el establecimiento de una relación amorosa que se configura como modelo de las relaciones de confianza subsiguientes pues, siguiendo a Nussbaum (2014), la confianza es un sentimiento que posibilita la emergencia de una relación de amor en la que la característica fundamental es el desarrollo de un interés genuino por el bienestar del otro.

Así, el sentimiento de confianza desde un punto de vista moral se vincula con la ética del cuidado (Niño, 2009). La ética del cuidado sigue 
los planteamientos de Caroll Gilligan, y se centra en el vínculo con el otro y el reconocimiento de la responsabilidad hacia los otros, por lo que representa una forma de moralidad que se fundamenta en el mundo relacional (Bedoya-Hernández, 2013).

En este sentido, la filósofa Annette Baier (1986), considera que la confianza pone el acento en una ética del cuidado y el amor, separándose de las éticas de la obligación, en tanto la primera se gesta en las relaciones familiares como caso paradigmático del vínculo moral al permitir la génesis de la cooperación social, y se caracterizan por ser íntimas y requerir de otro tipo de compromisos para mantenerlas diferentes a los del contrato social entre ciudadanos; en cambio, las éticas de la obligación ubican el modelo contractual en el centro de las relaciones humanas, y en ésta, la moral es entendida en términos de obediencia a unas leyes universales de libertad y justicia. Desde esta perspectiva, "confiar implica contar con la competencia y la buena voluntad de otros para cuidar y evitar dañar los bienes más preciados que hemos puesto en sus manos" (Calvo, 2009, p. 47).

En la misma línea, Niño (2010), retoma la confianza desde la perspectiva de la filósofa feminista Annette Baier y la teoría de comunidades morales de Rorty. Para la autora, la moralidad comienza en las relaciones recíprocas de confianza en los círculos o grupos íntimos como prerrequisito fundamental para integrar progresivamente a los extraños en la comunidad moral. Argumenta además que la moral comienza en el seno familiar, que acoge al recién nacido y le brinda afecto y amor, y de esta manera se van tejiendo lazos de confianza recíproca. Esta primera experiencia de amor es fundamental para poder desarrollar la capacidad de interés por el otro e ir ampliando el círculo de las relaciones de confianza, pues "responsabilizarse por el otro depende de la posibilidad de constituir comunidades morales más incluyentes" (Niño, 2010, p. 96). De esta manera, se entiende que las relaciones de confianza se gestan en las relaciones de amor, que posibilitan la emergencia del interés y la responsabilidad por el otro.

Es reconocido, entonces, que las experiencias tempranas de contacto, cuidado e interacción son fundamentales en el desarrollo afectivo y por ende en el desarrollo de sentimientos morales y en la emergencia de la moralidad en los niños (Narváez, 2010). Sin embargo, los escenarios educativos institucionalizados, como posibilidad de interacción con otros y ampliación del mundo relacional, también juegan un papel fundamental en el desarrollo moral de los niños. 
En los contextos educativos, se han elaborado diferentes modelos de educación moral y propuestas interventivas que le apuestan al desarrollo de habilidades y competencias morales para la toma de decisiones y que últimamente hacen un énfasis especial en el desarrollo de sentimientos morales (Escámez Sánchez, 2003; B Maxwell \& DesRoches, 2010; Bruce Maxwell \& Reichenbach, 2007; Moya, 2012). Sin embargo, no se encontraron investigaciones que abordaran la confianza, como sentimiento moral, en contextos de educación musical.

Se reconoce el lugar de la formación artística en el desarrollo infantil, pues se ha argumentado que las artes juegan un papel fundamental en la educación afectiva y por tanto, en la formación de la sensibilidad moral (Carr, 2005; Kroflič, 2012). Las artes cultivan la capacidad de imaginación narrativa, que se refiere a la capacidad de pensar cómo sería estar en el lugar de otra persona, de interpretar su relato y entender sus sentimientos, deseos y expectativas (Nussbaum, 2005).

La música, por su parte, está profundamente relacionada con la vida emocional, por las propiedades expresivas de la propia música y las emociones del oyente (Nussbaum, 2008). Está también arraigada a las prácticas culturales y circula como producción subjetiva de carácter simbólico y emocional (González Rey, 2008). El contexto de educación musical se erige entonces como un espacio de construcción subjetiva, en el que se ponen en juego procesos que van configurando la vida emocional y moral de los sujetos. Por tanto, comprender los procesos implicados en la construcción de la confianza, como sentimiento moral, que se desarrolla en el proceso de formación artística musical, es una posibilidad de acercarse al desarrollo moral y afectivo situándolo en las prácticas educativas.

Esta investigación tuvo como objetivo comprender las experiencias confianza en el proceso de educación musical de los niños y niñas de la Escuela Musical Amadeus. Se buscó, en un primer momento, relatar las experiencias de confianza en el proceso de educación musical y después, describir e interpretar el sentimiento de confianza en las relaciones con otros establecidas durante dicho proceso.

\section{Metodología}

Este estudio está fundamentado en el paradigma constructivista (Guba \& Lincoln, 2000); el enfoque de investigación es el interpretativo (2000), y 
se adoptó el método fenomenológico-hermenéutico (Van Manen, 2003). La investigación se realizó con los niños y niñas de la Escuela Musical Amadeus, una escuela de educación musical privada de la ciudad de Medellín. Para la selección de los participantes se realizó un muestreo por fases, que se correspondieron con los momentos de la investigación y las técnicas a utilizar.

En la primera fase se realizaron sesiones de fotohistoria (Garcia Chacon, González Zabala, Quiroz Trujillo, Velasquez Velasquez, \& Ghiso Cotos, 2002), como técnica interactiva, con los niños, niñas y adolescentes pertenecientes a cuatro agrupaciones instrumentales y corales de la academia, con el objetivo de identificar los sentimientos que ellos reconocían que habían emergido a lo largo del proceso de formación musical3. Los sentimientos que fueron mencionados con mayor frecuencia por los participantes fueron: pena, nervios, felicidad, miedo, confianza, rabia y tranquilidad.

En la segunda fase se realizaron sesiones de Fotolenguaje (GarciaChacon et al., 2002), para indagar por los sentimientos morales seleccionados a partir de la primera fase: confianza, felicidad, rabia, nervios y pena ${ }^{4}$. Para este momento los participantes fueron niños y niñas que habían participado en la fase anterior, que llevaban más de dos años en el proceso de educación musical.

Después de la codificación se eligieron los sentimientos de pena y confianza para ser profundizados en la siguiente fase, por la frecuencia de aparición en los relatos de los participantes, por ser significativos en la experiencia de educación musical y por la estrecha relación que se encontró entre los códigos que emergieron sobre ambos sentimientos. En este artículo se presentan los hallazgos relativos al sentimiento de confianza.

3. Dado que los trabajos grupales se realizaron en el horario y lugar de ensayo de las agrupaciones, y que en una misma agrupación había presencia tanto de niños como de adolescentes, el criterio de edad se flexibilizó para las dos primeras fases de la investigación, en las que se tenía como objetivo la identificación de los sentimientos morales que se presentaban con mayor frecuencia e intensidad en la experiencia de educación musical de los participantes. Para la última fase, en la que ya se había tomado la decisión de indagar sobre la experiencia de pena y confianza, la selección muestral fue más específica y homogénea, y los participantes fueron exclusivamente niños y niñas entre 9 y 11 años.

4. La pena fue entendida en este estudio como vergüenza, y ambos términos fueron tratados como sinónimos para efectos de la discusión de antecedentes investigativos y teóricos, según la acepción que trae la RAE en el uso que se le da al término en América Central, Las Antillas, Colombia, México, Panamá y Venezuela. Sin embargo, para la comprensión de la experiencia de los niños se hizo referencia al término con que ellos dan cuenta de su sentimiento: la pena, entendiendo que, en sus narraciones, los términos no son tratados como equivalentes, y que indagar sobre la vergüenza no fue un objetivo de esta investigación. 
En la tercera y última fase, los participantes fueron cuatro niños (dos varones y dos mujeres) entre 9 y 11 años, que habían participado de las fases anteriores. Se realizaron dos entrevistas en profundidad con cada uno. Como estrategia de triangulación se realizó un grupo focal, que se centró en el proceso de emergencia de la relación de confianza y se realizó con los cuatro participantes de las entrevistas en profundidad.

Se retomaron los procedimientos de análisis de datos de la teoría fundamentada, como el microanálisis, la codificación y la categorización (Strauss \&Corbin, 2002). El proceso de codificación se hizo mediante el microanálisis para generar categorías iniciales y para sugerir relaciones entre ellas. Se utilizó como instrumento para el análisis de los datos el software Atlas TI en la versión 7. Las consideraciones éticas del estudio estuvieron basadas en la Resolución número 8430 de 1993 del Ministerio de Salud de Colombia, por la cual se establecen las normas científicas, técnicas y administrativas para la investigación en salud y se solicitó a los participantes firmar el asentimiento informado y a sus representantes legales el consentimiento informado.

\section{Resultados}

\section{La confianza en el proceso de enseñanza-aprendizaje}

En la experiencia de educación musical, los participantes describen la confianza como parte de los procesos de enseñanza-aprendizaje de la música. El hecho de enseñar las técnicas de ejecución de un instrumento o del canto, llevado a cabo por los profesores, es reconocido por los participantes como un acto generador de confianza porque, según sus palabras, les brinda la posibilidad de mejorar y avanzar en la interpretación del instrumento o el canto. Ellos sienten más confianza a medida que aprenden "cosas nuevas" $\left(\mathrm{P}_{1}\right)$ y van progresando en el proceso musical. A su vez, el profesor se configura como una persona beneficiaria de confianza porque "enseña" y, en este sentido, la acción misma de enseñar le confiere características confiables, pues se vincula con las cualidades de un agente de cuidado (dar consejos, ayudar, enseñar buenas prácticas, corregir y cuidar del daño). Otra característica que se identifica en sus narraciones sobre una persona confiable es la manera de amonestar. Los niños sienten confianza cuando saben que la profesora los corrige sin gritos, "con palabras lindas, con calma" ( $\mathrm{P} 2), \mathrm{y}$ de una manera que permita el aprendizaje. 
"También que la profe nos brinda mucha confianza, en que nos dice que respiremos, por ejemplo que no prendamos el ventilador porque nos daña la voz, como que ella nos ayuda mucho, nos da consejos de cómo cantar." $\left(\mathrm{P}_{2}\right)$

Hay una diferencia clara en los relatos sobre los ensayos y las clases, en contraste con las situaciones en las que se presentan en público. Por ejemplo, es recurrente escucharles contar su preocupación ante la posibilidad de equivocarse en las presentaciones en público, situación que no sucede en los espacios de ensayo y clase, porque allí los niños saben que tienen la posibilidad de repetir las canciones, y este escenario es reconocido por ellos como una oportunidad para el aprendizaje, tal como se evidencia en el siguiente fragmento:

"yo me doy las oportunidades de equivocarme en el ensayo.

Entonces para mí esa es como un oportunidad que le dan a uno una y mil veces." $\left(\mathrm{P}_{4}\right)$

De esta manera, el ensayo es visto por los niños como una oportunidad para corregir el errory realizar una buena ejecución, a diferencia del espacio de presentación en público. El escenario de ensayo se configura entonces como un espacio de aprendizaje, en el cual el proceso es valorado más que el resultado.

\section{Relación con el público:} cuando en el público hay personas conocidas.

Estar en el escenario durante un concierto configura una situación emocional intensa para los niños, en la cual la confianza puede estar o no presente dependiendo de las personas que asisten, del tipo de relación que sostienen con ellas, y de la valoración que hacen de dichas personas. Las personas que asisten al concierto como público son determinantes en la manera como ellos experimentan el sentimiento confianza durante su presentación. En este sentido, los niños hacen una distinción clara entre las personas conocidas y desconocidas.

Se encontró que cuando los niños están con personas que conocen, el sentimiento de confianza prevalece, mientras que, cuando el público está conformado mayoritariamente por personas desconocidas, la confianza disminuye y aparece el sentimiento de pena. 
"pero por ejemplo ya en el de Comfama o los conciertos que hacen aquí la pena como que baja, pues, uno se siente como más tranquilo porque es gente que uno ya conoce, uno es sociable con ellos"( $\left.\mathrm{P}_{1}\right)$

Además, se advierte en el relato de los niños una preocupación e incertidumbre constante por el gusto o disgusto del público por su presentación. Los niños miran el rostro, los gestos y las reacciones del público para leer si les está gustando la interpretación. Cuando ven sonrisas, aplausos y halagos sienten que lo están haciendo bien y, en consecuencia, el sentimiento de confianza aumenta, mientras que, cuando creen que no les está gustando el sentimiento que prevalece es la pena.

"Uno ya mira a la gente, ve que les está gustando, están sonriendo y todo eso, uno lo que más le importa es que a los papás les guste y que a la gente también" ( $\left.\mathrm{P}_{2}\right)$

En general, ser reconocido por el público es alentador para los participantes y genera un sentimiento de confianza. Sin embargo, cuando el reconocimiento proviene de sus padres o familiares, de quienes reciben manifestaciones de cariño como besos y abrazos y expresiones de aliento y admiración, adquiere mayor importancia para los niños. Así, cuando dichas expresiones provienen de personas significativas como los padres, revisten un valor especial para los niños y son determinantes en el aumento de la confianza y la consecuente disminución de la pena.
"Ese día del concierto mi tía me dijo, pues yo no sé, ella me gritó y me dijo "iNo importa Juan5, siga, siga! Usted está tocando muy bien", entonces ahí sentí como un poquito, como que se me quitó la pena" $\left(\mathrm{P}_{1}\right)$
"Por ejemplo yo salgo del escenario y mis papás van a recogerme, pues ellos me abrazan y todo, y dicen que toqué muy bien, ahi se... pues, se va todo eso, pues, eso desaparece [la pena]."( $\left.\mathrm{P}_{1}\right)$

En definitiva, se advierten experiencias afectivas diferenciales cuando los niños se presentan ante personas conocidas y desconocidas. Con el desconocido, el sentimiento de pena es más intenso; mientras que, con las personas conocidas, el sentimiento de confianza prevalece, pues 
de ellas se espera comprensión, empatía, apoyo, palabras de aliento y reconocimiento.

\section{Estrategias de regulación emocional}

Los participantes identifican el sentimiento de confianza como un sentimiento posibilitador de un mejor desempeño musical, que les ayuda a hacerle frente al sentimiento de pena que se presenta en momentos de tensión y que, por el contrario, impide una buena interpretación en el escenario. Los niños hacen referencia a algunas estrategias que ponen en acción cuando sienten pena; dichas estrategias les permiten sentir confianza en los momentos previos y durante las presentaciones $y$, por tanto, les ayuda a evitar las consecuencias indeseables que ellos asocian a la experiencia de pena (paralizarse y equivocarse).

Una de las estrategias emprendidas por los niños consiste en dirigirse a sus amigos o familiares, acercarse y conversar con ellos. Las conversaciones con personas cercanas producen calma en situaciones de tensión como el momento previo a un concierto o la presentación misma. Los niños también narran que cuando se equivocan y sienten pena desearían bajar del escenario y buscar a sus padres.

\section{"Pues yo hablo con mis amigos, si no tengo tanta confianza yo me pongo a hablar con mis amigos y así se me quita un poquito la pena y el miedo" $\left(\mathrm{P}_{3}\right)$}

El movimiento puede ir del niño hacia quienes le profieren calma, o ser emprendido por otros, que identifican el sentimiento de pena y se dirigen a calmarlos. Esta acción puede ser iniciada por los docentes, los padres o los mismos compañeros. Inclusive, los participantes refieren que cuando ven que uno de sus compañeros está sintiendo pena, sienten pesar y desearían ayudarlo, en ocasiones indicándole cómo hacerle frente a la pena ("le dan ganas como de uno ayudarlo a quitar esa pena", P2).

P2: pero la profe nos brindó mucho apoyo durante ese día

$\mathrm{E}:$ ¿Que hacía la profe ese día?

P2: Ella nos trataba de calmar, nos decía que todo iba a salir bien y ya.

En definitiva, estas estrategias de regulación emocional son movimientos emprendidos por los niños o por otros, para hacerle frente al 
sentimiento de pena y se caracterizan por la búsqueda de proximidad con las personas significativas, pues en las narraciones de los participantes se evidencia que buscan acercarse a las personas depositarias de confianza, quienes les profieren calma a través de las conversaciones.

\section{"Coger confianza": afianzar la confianza en una relación}

Una relación de confianza es resultante de un proceso en el cual el sentimiento de confianza se va afianzando paulatinamente hasta convertirse en una característica fundamental de la relación interpersonal. Los niños llegan a construir relaciones de confianza con sus compañeros y profesores de la escuela musical, que son reconocidas por ellos mismos como "relaciones de confianza", y a su vez, narran haber atravesado por un proceso de consolidación de dicha relación en el que hacen un tránsito desde el sentimiento inicial de pena, hasta la confianza. Inicialmente, cuando los niños llegan por primera vez a la escuela de música y no conocen el espacio, ni a sus compañeros y profesores, ellos refieren un sentimiento de extrañeza e incomodidad, que los niños asocian a la pena, y que es, en definitiva, desagradable. Ese sentimiento se presenta ante una situación que es totalmente novedosa para los niños.

\section{"sí, porque ya uno como que va cogiendo más confianza con el profesor y ya como que uno se siente mejor, porque yo al principio sí me sentía como extraño." ( $\left.\mathrm{P}_{2}\right)$}

Los participantes narran que antes de llegar por primera vez a un grupo se hacen imágenes sobre lo que podría pasar y la manera como pueden ser tratados por otros. El sentimiento de pena en las primeras interacciones es explicado por los niños por la posibilidad de que las personas que apenas van a conocer los rechacen, hagan juicios sobre ellos, o sean ambivalentes en el trato. En este caso, el trato que pueden recibir de las personas que no conocen es inesperado y puede resultar amenazante y agresivo.

"De que esa persona no le cayera bien yo, eh, o que no quisieran ser mis amigos, eso es lo que me daba más pena, o que fueran mis amigos y al tiempo me humillaran y me dijeran cosas que..." (Grupo focal, $\mathrm{P}_{4}$ )

En vista de que los niños llegan generalmente a un grupo que ya está conformado y del cual no hacen parte aún, la pena es un sentimiento 
prevalente en ese momento y los niños emprenden estrategias para hacerle frente y poder acercarse a los otros integrantes, lo que les exige tomar la iniciativa para comenzar una interacción emitiendo una sonrisa o iniciando una conversación, para poder después acercarse a otros niños. La intervención de los profesores también es decisiva en este proceso acogiendo a los nuevos integrantes, distensionando el ambiente con un chiste y motivando a los otros a que también hagan lo mismo.

"yo para quitar la pena o algo así, yo digamos que aquí está alguien, entonces yo me reía y empezaba ya después a hablar con él, entonces ya empezaba a hablar con otros." (Grupo focal, P3)

En la primera interacción, los niños resaltan la importancia de la amabilidad en una persona como necesaria para comenzar a entablar una relación de confianza. La confianza puede ser tanto una cualidad de la relación como una cualidad de la persona que es valorada como una persona confiable o no y, para los niños, una persona amable es una persona confiable yes, a su vez, valorada como una buena persona. Saludar, sonreír y hablar entre sí son señales de amabilidad o trato amigable como posibilitador de una relación de confianza, que se lee tanto en el otro como en sí mismo, configurándose también como una necesidad o un deber ser en la relación con los otros, pues "ser una persona buena gente" permite la emergencia de una relación de confianza.

"como que uno llegue y lo salude a uno, o también uno también ser una persona buena gente para que los demás también tengan confianza en uno" (P2)

La relación de confianza es narrada como una relación recíproca, en la que las acciones que generan, afianzan e indican confianza o no, son realizadas por todos los actores de la relación. Los niños permanentemente leen las acciones de los otros, las interpretan, las valoran y actúan en consecuencia; y de la misma manera, emprenden acciones amables como sonreír, conversar o ayudar, esperando que el otro también responda de manera similar.

"uno también ser una persona buena gente para que los demás también tengan confianza en uno, porque uno tampoco tiene que esperar algo de los demás, sino que también uno también tiene que dar." ( $\left.\mathrm{P}_{2}\right)$ 
El proceso de coger confianza comienza con el desconocimiento absoluto del otro, pasando por una serie de interacciones que permiten ir conociéndolo paulatinamente, hasta llegar a establecer una relación de amistad, que es la relación de mayor confianza. Para los niños, mientras más aspectos se conozcan del otro, mayor es la confianza y, a mayor confianza, más se consolida la relación, que puede ir mejorando en su calidad hasta llegar a ser muy buenos amigos.
"Sí, porque desde que uno empieza, uno no las conoce, entonces uno no tiene tanta confianza, pero ya cuando va pasando, pasando, ya uno tiene más confianza, y ya al final ya tiene mucha confianza, y ya pueden que sean muy buenos amigos" $\left(\mathrm{P}_{3}\right)$

Los participantes refieren que las experiencias que han compartido en conciertos, ensayos y presentaciones, van afianzando la relación de confianza. Con frecuencia recuerdan episodios de experiencias compartidas, cuentan anécdotas y recuerdan situaciones graciosas de las que posteriormente se ríen. Resaltan además que el tiempo es un factor clave en el establecimiento de una relación de confianza. El transcurrir del tiempo, al igual que la interacción frecuente, permite ir afianzando la confianza.

"No sé, como el tiempo, que uno pase más tiempo con ellos, las experiencias que ha vivido, las presentaciones también son mucha confianza porque entre uno más pase tiempo con ellos más confianza tiene, y también vamos a tener anécdotas qué contar, pues más adelante..." $\left(\mathrm{P}_{2}\right)$

La confianza es además un sentimiento que tiene un proceso lento y paulatino de aparición que requiere de acciones de ambas partes para instalarse en la relación. Según el relato de los niños, la confianza se gana, como se evidencia en el siguiente fragmento: "[El profe] se ha ganado nuestra confianza pedacito a pedacito" ( $\left.\mathrm{P}_{4}\right)$. En este sentido, los participantes resaltan las acciones de ayuda y colaboración como elementos fundamentales en las relaciones de confianza. Prestar el folder o la partitura a quien la olvidó, compartir comida con alguien que no llevó, ayudar a solucionar un problema o acompañar a aprender una canción a quien no la sabe, son acciones que ellos identifican como generadoras de confianza y como indicadores de que en una relación hay confianza. 
Los niños acuden a las personas con quienes sienten confianza para contarles sus problemas, constatando que les ayudan a darle solución. El no recibir ayuda genera rabia y limitaciones para entablar una relación de confianza. Es importante resaltar que ellos consideran que pueden encontrar ayuda en las personas de la academia cuando la requieren.

"Es que aquí [en la academia] todo el mundo te va a dar la mano para ayudarte, y eso" ( $\left.\mathrm{P}_{2}\right)$

Así mismo, esperar a recibir ayuda de las personas en quienes confían, se vincula con una expectativa de incondicionalidad. Los niños refieren que una de las características de las relaciones de confianza es que pueden contar con estas personas en todo momento, especialmente cuando se encuentran en una situación problemática y requieren del apoyo y la colaboración de esa persona en quien confían. Los niños esperan entonces que estas personas no sólo los acompañen en los momentos de juego, risas, chistes y conversaciones cotidianas, sino que también estén disponibles cuando ellos los requieran, y el hecho de no hacerlo puede llegar a fracturar la relación de confianza.

"Ayudándole a hacer cosas, en algún problema que tengan, ya, como estar al lado de ellos siempre, como que uno se ría con ellos, pero tampoco es reírse sino también estar en las malas" $\left(\mathrm{P}_{2}\right)$

En la misma línea, tres acciones se configuran como indicadores clave en la valoración de la calidad e intensidad del sentimiento de confianza en una relación: la posibilidad de contar intimidades, la consecuente certeza de que el otro guardará sus secretos y el cumplimiento de promesas. Estas acciones afianzan la relación de confianza y, no hacerlo, fractura a su vez la confianza. Los niños reportan que, inicialmente, contar situaciones de la vida íntima es un riesgo que ellos deciden correr, y en ese momento ponen a prueba al otro en su capacidad de guardar secretos. Ellos reportan que quien lo guarda, es considerado como un "verdadero amigo", y tener la posibilidad de contarlo y confiar en el otro genera un sentimiento liberador y tranquilizante.

"Con el paso del tiempo (...) uno por ejemplo se arriesga a contarle a ellos a ver si sí son de confianza o no, entonces ahí es donde uno se da cuenta de eso." ( $\left.\mathrm{P}_{1}\right)$

$\mathrm{E}:$ ¿Y cómo sería un buen amigo? 


\section{P: Que sea bueno, pues, que cumpla todo lo que le promete a uno $\left(\mathrm{P}_{3}\right)$}

En definitiva, se reconoce que el sentimiento de confianza tiene un proceso de aparición lento y paulatino, y se va "cogiendo" a partir de una serie de interacciones que posibilitan su emergencia. Dicho proceso comienza con el encuentro con otro desconocido que inicialmente resulta amenazante y genera pena, y se va consolidando como una relación de confianza a partir de experiencias compartidas y acciones emprendidas por ambas partes de la relación, comenzando por gestos de amabilidad, pasando por acciones de ayuda y cooperación, hasta llegar a establecer una relación de amistad, que es la relación de mayor confianza, que está asociada a una expectativa de incondicionalidad y en ella existe la posibilidad de contar intimidades y la certeza de que el otro guardará los secretos y cumplirá las promesas.

\section{Discusión}

Desde la experiencia de los niños, el sentimiento de confianza se reconoce como una característica fundamental en una relación interpersonal que se configura como una "relación de confianza". Se encuentra fuertemente vinculado a las acciones de cuidado (dar consejos, ayudar, enseñar buenas prácticas, posibilitar y promover el aprendizaje y las nuevas experiencias, corregir y cuidar del daño). En este sentido se acerca a las teorizaciones que vinculan la confianza, desde el punto de vista moral, con la ética del cuidado (Baier, 1986; Niño, 2009)

En la experiencia de aprendizaje musical, los niños describieron sostener relaciones de confianza con sus familiares, profesores, amigos y compañeros de la academia y en todas estas relaciones se evidencian acciones de cuidado que son emprendidas por diferentes sujetos de la relación. Es importante destacar el lugar que ocupan los padres y maestros en la experiencia de educación musical de los niños, especialmente en momentos de tensión como las presentaciones musicales, en los que cumplen funciones de un agente de cuidado. Así mismo, los niños van estableciendo relaciones de confianza con sus pares (compañeros y amigos), en las que ellos también cuidan del otro.

Para Calvo (2009), la confianza es un concepto excluido del corpus de las teorías morales que, ciertamente, está involucrado en la ética del 
cuidado y del amor. En esta misma vía, Martha Nussbaum (2014) reconoce la confianza como un sentimiento fundamental en la emergencia de una relación de amor cuya característica fundamental es el desarrollo de un interés genuino por el otro. A partir de las narraciones de los niños se evidenció que en las relaciones de confianza ellos emprenden acciones de cuidado que connotan un interés por el otro pues, para los niños, lo que le sucede a sus compañeros como sentir pena, no saberse una canción, haber olvidado las partituras, equivocarse, estar preocupado, ser burlado por otros o vivir una situación problemática, son situaciones de interés e incumbencia no solo para quien las vive, sino para las personas con quienes han establecido una relación de confianza y, por tal motivo, se emprenden acciones de ayuda y cuidado.

El sentimiento de confianza, siguiendo a Nussbaum (2014), es entendido como un sentimiento que se encuentra en profunda relación con el amor. La autora entiende el amor como "un reconocimiento placentero del otro como un ser valioso, especial y fascinante" (p. 214). El desarrollo de una preocupación e interés genuino por el otro implica que el sujeto desarrolle un sentimiento de confianza que tiene lugar a partir de los interjuegos en las relaciones tempranas, y es posible por el comportamiento amable del padre y/o madre, lo que permite la consolidación de una relación amorosa que impulsa al niño hacia una toma de perspectiva empática. El amor permite hacerle frente al sentimiento de vergüenza que se relaciona con el reconocimiento de la propia indefensión e impotencia humanos (Nussbaum, 2006).

El sentimiento de confianza se consolida entonces en las relaciones tempranas y se continúa a lo largo de la vida manifestándose tanto de manera lingüística como pre-lingüística, a través de sonrisas, abrazos, besos, miradas, aplausos y halagos. Desde la experiencia de los participantes, todas estas manifestaciones, cuando provienen de figuras significativas para el niño, cobran especial relevancia en el fortalecimiento de la confianza y disminución de la pena.

La forma prototípica de cuidado es el vínculo de apego entre el bebé y su madre como la desarrollaron Bowlby y Ainsworth, y los desarrollos teóricos al respecto se han centrado en los primeros momentos del desarrollo y en las relaciones con los cuidadores primarios como precursoras y basamento de las relaciones interpersonales subsiguientes (Fonagy, 1999; Lecannelier, 2004; Stern, 2003), prestando especial atención a la constitución intersubjetiva del sí mismo; sin embargo, 
"todo esto atañe a las relaciones de intimidad en edades tempranas, pero la misma dinámica se reproduce en diferentes fases evolutivas de la personalidad" (Nussbaum, 2014, p.215) y, en este sentido, el cuidado se aprende y es la base de la identidad del sujeto y de su proceso de desarrollo (Bedoya-Hernández, 2013).

El sentimiento de confianza se configura como cualidad fundamental de una relación interpersonal que es reconocida por los sujetos como "relación de confianza". Dicha relación, desde la experiencia de los niños, tiene algunas características que la consolidan como una relación de cuidado:

En primer lugar, posibilita el aprendizaje y el acceso a nuevas experiencias $y$, de esta manera, la relación misma y el espacio en que se desarrolla esta relación se configuran como una oportunidad para explorar y construir nuevos conocimientos, pues "si se produce el aprendizaje, será siempre como un efecto emergente de la relación entre los participantes" (Vásquez \& Escámez, 2010, p. 12). Las relaciones de confianza que se gestan en el espacio del ensayo y la percepción del mismo como un espacio seguro que posibilita el aprendizaje, son las razones por las cuales el ensayo es identificado por los niños como un espacio de confianza.

En segundo lugar, la relación de confianza es una relación que promueve y facilita la regulación emocional y, de esta manera, el agente de cuidado está en la capacidad de proferir calma en momentos de angustia y tensión, esto es, identificar la angustia y dirigirse al niño para calmarlo; de igual manera, los niños sienten que pueden acudir a la persona en quien confían cuando lo requieren. Investigaciones sobre el desarrollo afectivo infantil han puesto especial énfasis en el sistema de cuidado como regulador emocional. En este sentido, Fonagy (1999), ha identificado la regulación afectiva como la capacidad del cuidador de modular las respuestas afectivas del infante, lo que posibilita posteriormente en el niño el desarrollo de la capacidad de autoregularse (Stern, 2003). Se evidenció que para los niños, la relación con otros, que está cargada de afecto, es reguladora del sentimiento de pena, sentimiento que ellos identifican como indeseable porque paraliza e imposibilita la adecuada interpretación musical.

En tercer lugar, la relación de confianza es considerada como una relación empática y comprensiva, en la que la persona depositaria de 
confianza está en la capacidad de comprender los sentimientos del niño. Los sentimientos comprensivos están a su vez relacionados con conductas de ayuda y colaboración (Nussbaum, 2010). De esta manera, la relación amorosa impulsa a la reciprocidad y a la toma de perspectiva empática que hace que la acción sea expresión de un interés genuino por el otro (Nussbaum, 2014).

Por último, la relación de confianza está asociada a una expectativa de incondicionalidad pues se espera que el otro responda a las necesidades propias y que esté disponible cuando se le solicite, no solo "en las buenas", sino también en los momentos de dificultad. La disponibilidad ha sido identificada por las teorías del apego como un rasgo fundamental en una relación de cuidado que es promotora de un adecuado desarrollo psicoafectivo en el niño (Sánchez, 2011).

Estas características no son exclusivas de las relaciones parentofiliales, pues los niños entre ellos establecen relaciones de cuidado y emprenden acciones, que se corresponden con este tipo de relación, con sus pares (compañeros y amigos). Los niños amonestan a quien se burla a manera de defensa del otro, piden ponerse en su lugar, consuelan y ayudan a sus compañeros a disminuir la pena; todas estas se consolidan como acciones de cuidado a las cuales subyace el interés por el otro. El proceso de "coger confianza", implica que los niños van ampliando el círculo de sus relaciones de confianza (Niño, 2010), incluyendo en él a otros que antes eran desconocidos y frente a quienes sentían pena.

En este sentido, se evidencia que el amor, el interés, el cuidado y la responsabilidad por el otro no surgen como una obligación o imposición, antes bien, se desarrollan a partir de relaciones de confianza que se van afianzando y que posibilitan la emergencia de otras relaciones de confianza. Para Nussbaum (2014), "la confianza no puede nacer de la aplicación de unas reglas de equidad exclusivamente; en el fondo, bien poco tiene que ver con tales reglas" (p. 214). Por el contrario, se gesta en una relación amorosa de la cual surge la moralidad en el niño, dado que le brinda la posibilidad de sentirse amado y cuidado para poder amar y cuidar del otro.

La confianza se concibe como un proceso, que comienza a gestarse en el círculo más íntimo al que el sujeto pertenece, la familia, y que se va ampliando a medida que se va incluyendo en él a otros que anteriormente eran extraños. Se destaca entonces el carácter procesual de la confianza 
al ser un sentimiento que se "gana", se "va cogiendo", se consolida y se mantiene, pero que también puede fracturase, vulnerarse y perderse. En este orden de ideas, el sentimiento de confianza encierra en su proceso varias acciones, siendo el mismo sentimiento también una acción: confiar, lo que destaca su carácter activo, dinámico y procesual.

Si se considera que el sentimiento de confianza se gesta progresivamente en las nuevas relaciones de los sujetos, y que, con cada nueva relación de confianza se va ampliando el círculo relacional del niño (Niño, 2010), el sentimiento de confianza se entiende como un sentimiento de inclusión, pues implica el interés y preocupación genuina por personas que inicialmente eran extrañas para el niño y que ahora son acogidas y están siendo incluidas en su grupo íntimo de relaciones de confianza. La inclusión supone un movimiento de acercamiento producto de la iniciativa de los sujetos implicados en la relación que, aunque no se conozcan, emprenden acciones como sonreír, saludar o hacer un chiste, para conocerse $y$, posteriormente, confiar. La inclusión progresiva de una persona en el grupo íntimo de relaciones de confianza implica su reconocimiento como sujeto y agente de cuidado, pues con esa persona se emprenden acciones de cuidado y de ella se esperan actitudes de reciprocidad en la relación.

\section{Referencias bibliográficas}

Baier, A. (1986). Trust and antitrust. Ethics, 96(2), 231-260.

Bedoya-Hernández, M. (2013). Redes del cuidado: Ética del destino compartido en las madres comunitarias antioqueñas. Revista Latinoamericana de Ciencias Sociales, Niñez y Juventud, 11(2), 741-753.

Calvo, A. (2009). La confianza: ¿categoría mediadora entre la obligación y el cuidado en filosofía moral? La voz de Annette Baier en la filosofía feminista. Universitas Philosophica, 53(26), 37-53.

Camps, V. (2011). El gobierno de las emociones. Barcelona: Herder.

Carr, D. (2005). On the contribution of literature and the arts to the educational cultivation of moral virtue, feeling and emotion. Journal of Moral Education, 34(2), 137-151. 
Escámez Sánchez, J. (2003). Pensar y hacer hoy educación moral. Teoría de la educación, 15, 21-31.

Fonagy, P. (1999). Persistencias transgeneracionales del apego: una nueva teoría. Aperturas Psicoanalíticas: Revista Internacional de Psicoanálisis, 3. Recuperado a partir de http://www.aperturas.org/articulos.php?id=0000086\&a=Persistenciastransgeneracionales-del-apego-una-nueva-teoria

Garcia Chacon, B. E., Gonzalez Zabala, S. P., Quiroz Trujillo, A., Velasquez Velasquez, A. M., \& Ghiso Cotos, A. M. (2002). Técnicas interactivas para la investigación social cualitativa. Medellín: Fundación Universitaria Luis Amigo.

González, J. (2000). El paradigma interpretativo en la investigación social y educativa. Cuestiones Pedagógicas. Revista de Ciencias de la Educación, 15, 227-246.

González Rey, F. L. (2008). Psicología y arte: razones teóricas y epistemológicas de un desencuentro. Tesis Psicológica, 3, 140-159.

Guba, E., \& Lincoln, Y. (2000). Paradigmas en competencia en la investigación cualitativa. En C. A. Denman \& J. A. Haro (Eds.), Por los rincones: antología de métodos cualitativos en la investigación social. Hermosillo: El Colegio de Sonora.

Jiménez Mendoza, W., Soto Carrión, C., \& Urrutia Huamán, R. (2015). Relación entre modelo PIHEM y gestión educativa en instituciones educativas del nivel inicial en el distrito de Abancay Apurímac Perú, 2014. Revista De Investigaciones UNAD, 14(1), 81 95. doi:http://dx.doi.org/10.22490/25391887.1347

Kroflič, R. (2012). The role of artistic experiences in the comprehensive inductive educational approach. Pastoral Care in Education, 30(3), 263-280. http://doi.org/10.10 80/02643944.2012.671342

Langer, E. (2016). La construcción de confianza para el estudio de prácticas de resistencia en la escolarización de jóvenes en contextos de pobreza urbana. Revista Interamericana de Investigación, Educación y Pedagogía, 9(2), 113-137.

Lecannelier, F. (2004). Los aportes de la Teoría de la Mente (ToM) a la Psicopatología del Desarrollo. Terapia Psicológica, 22(1), 62-67.

Maxwell, B., \& DesRoches, S. (2010). Empathy and social-emotional learning: Pitfalls and touchstones for school-based programs. En B. Latsko\& T. Malti (Eds.), Children's moral emotions and moral cognition: Developmental and educational perspectives. New Directions for Child and Adolescent Development (Vol. 129, pp. 33-53). San Francisco: Jossey-Bass.

Maxwell, B., \&Reichenbach, R. (2007).Educating moral emotions: a praxiological analysis. Studies in Philosophy and Education, 26(2), 147-163.

Moya, J. (2012). Las emociones y la toma de decisiones morales. Moralia, 35, 155-177. 
Narváez, D. (2010). The emotional foundations of high moral intelligence. En B. Latzko\& T. Malti (Eds.), Children's moral emotions and moral cognition: Developmental and educational perspectives. New Directions for Child and Adolescent Development (Vol. 129, pp. 77-94). San Francisco: Jossey-Bass.

Niño, A. (2010). Desde las interacciones participativas hacia las comunidades morales. Discusiones filosóficas, 15, 83-96.

Nussbaum, M. (2005). El cultivo de la humanidad: una defensa clásica de la reforma en la educación liberal. Barcelona: Paidós.

Nussbaum, M. (2006). El ocultamiento de lo humano: repugnancia, vergüenza y ley. Buenos Aires: Katz Editores.

Nussbaum, M. (2007). Los sentimientos morales y el enfoque de las capacidades. Claves de razón práctica, 169, 28-35.

Nussbaum, M. (2008). Paisajes del pensamiento: La inteligencia de las emociones. Ediciones Paidós Ibérica.

Nussbaum, M. (2010). Sin fines de lucro: por qué la democracia necesita de las humanidades. Madrid: Katz.

Sánchez, M. (2011). Apego en la infancia y apego adulto. Influencia en las relaciones amorosas y sexuales. (Trabajo fin de Máster). Universidad de Salamanca, Salamanca. Recuperado a partir de http://gredos.usal.es/jspui/bitstream/10366/99355/1/TFM_ EstudiosInterdisciplinaresGenero_SanchezHerrero_M.pdf

Stern, D. N. (2003). El mundo interpersonal del infante: una perspectiva desde el psicoanálisis y la psicología evolutiva. Buenos Aires: Paidós.

Silva Carreño, W., \& Beltrán Martin, J. (2015). El rol de género como fundamento humanista de la formación para la ciudadanía. Revista De Investigaciones UNAD, 14(1), 7 - 17. doi:http://dx.doi.org/10.22490/25391887.1343

Strauss, A. L., \&Corbin, J. M. (2002). Bases de la investigación cualitativa: técnicas y procedimientos para desarrollar la teoría fundada. Medellín: Editorial Universidad de Antioquía, Facultad de Enfermería de la Universidad de Antioquía.

Van Manen, M. (2003). Investigación educativa y experiencia vivida: ciencia humana para una pedagogía de la acción y la sensibilidad. Barcelona: Idea Books.

Vásquez, V., \&Escámez, J. (2010). La profesión docente y la ética del cuidado. Revista Electrónica de Investigación Educativa, Número especial. Recuperado a partir de http:// redie.uabc.mx/contenido/NumEsp2/contenidoverdera.html 